Acta Cryst. (1980). A36, 503-509

\title{
A Theoretical Study of Elastic X-ray Scattering
}

\author{
By ROBERT F. STEWART* \\ University of Groningen, Chemical Physics Laboratory, Neijenborgh 16, 9747 AG Groningen, The Netherlands \\ AND DIRK FEIL \\ Twente University of Technology, Chemical Physics Laboratory, PO Box 217, 7500 AE Enschede, \\ The Netherlands
}

(Received 21 July 1979; accepted 11 December 1979)

\begin{abstract}
Bragg X-ray scattering intensities are defined as scattering by the thermodynamic average electroncharge density. Purely elastic, kinematic X-ray scattering by a target in thermal equilibrium is always larger than Bragg scattering. At low temperatures, the elastic scattering becomes Bragg scattering. For large molecules, such as a crystal, at ordinary temperatures the elastic and Bragg scattering differ in a relative sense by $O\left(N^{-1}\right)$, where $N$ is the number of vibrational degrees of freedom. For most practical cases the Bragg scattering is essentially the same as purely elastic scattering of $\mathrm{X}$-rays.
\end{abstract}

\section{Introduction}

In the past decade and a half, there has been an intensive effort to attempt detailed mapping of electrondensity distributions in crystals from accurately measured X-ray diffraction data. Several schools and conferences have been held on the subject in the last five years and reviews have also been written ( $c f$. Phys. Scr., 1977; Isr. J. Chem., 1977; Coppens \& Stevens, 1977). The reduction of the measured diffraction intensities to lines of constant electron density or to charge-density-dependent properties requires a large amount of physical theory. Since in X-ray scattering by a target in thermal equilibrium with its environment, a large number of quantum states of the scattering system are involved, statistical mechanics is called upon.

Apparently, in all treatments of crystal X-ray diffraction the structure factor is treated as a rather fundamental property of the crystal. It is defined as the Fourier transform of the thermal-average electron

\footnotetext{
* Permanent address: Department of Chemistry, Carnegie Mellon University, 4400-5th Ave., Pittsburgh, PA 15213, USA.
}

0567-7394/80/040503-07\$01.00 density in the crystallographic unit cell:

$$
F_{\text {Bragg }}(\mathbf{K}) \equiv \int_{\text {cell }} \overline{\rho(\mathbf{r})} e^{j \mathbf{K} \cdot \mathbf{r}} \mathrm{d}^{3} \mathbf{r},
$$

in which $\overline{\rho(\mathbf{r})}$ is the thermal average value of the electron density. The integrated intensity of a Bragg reflexion is then related to $F_{\mathrm{Bragg}}(\mathbf{K})$ with a variety of correction terms that include deviations from kinematic scattering theory such as absorption, extinction and multiple scattering and correction for thermal diffuse scattering. If the correction terms, the various constants, the Lorentz and polarization factors and the intensity of the incident beam are absorbed into the integrated intensity, then a reduced intensity, $I$, is equated to (e.g. Woolfson, 1970)

$$
I=\left|F_{\text {Bragg }}(\mathbf{K})\right|^{2} \equiv I_{\text {Bragg }}(\mathbf{K}) \text {, }
$$

in which $\mathbf{K} \equiv \mathbf{k}_{1}-\mathbf{k}_{0}$, the difference between the wavevectors of the diffracted and incident beam respectively.

In order to calculate $\overline{\rho(\mathbf{r})}$, the systems of the ensemble are chosen so as to replicate the thermodynamic state of the crystal.

The electron density corresponding to the state $|m\rangle$ of the crystal, consisting of $L$ electrons and $M$ nuclei, is given by

$$
\begin{aligned}
\rho_{m}(\mathbf{r})=L & \int \Psi_{m}^{*}\left(\mathbf{r}, \sigma_{1}, \mathbf{x}_{2}, \ldots \mathbf{x}_{L}, \mathbf{Q}_{1}, \ldots \mathbf{Q}_{M}\right) \\
& \times \Psi_{m}\left(\mathbf{r}, \sigma_{1}, \mathbf{x}_{2}, \ldots \mathbf{x}_{L}, \mathbf{Q}_{1}, \ldots \mathbf{Q}_{M}\right) \\
& \times \mathrm{d} \sigma_{1} \mathrm{~d}^{4} \mathbf{x}_{2} \ldots \mathrm{d}^{4} \mathbf{x}_{L} \mathrm{~d}^{4} \mathbf{Q}_{1} \ldots \mathrm{d}^{4} \mathbf{Q}_{M},
\end{aligned}
$$

in which $\mathbf{x}_{i} \equiv\left(\mathbf{r}_{i}, \sigma_{i}\right)$, the positional and spin coordinates of electron $i ; \mathbf{Q}_{j} \equiv\left(\mathbf{R}_{j}, \Sigma_{j}\right)$, the positional and spin coordinates of nucleus $j ; \mathbf{x} \equiv\left(\mathrm{x}_{1}, \ldots \mathrm{x}_{L}\right), \mathbf{Q} \equiv$ $\left(\mathbf{Q}_{1}, \ldots \mathbf{Q}_{M}\right), \mathbf{R} \equiv\left(\mathbf{R}_{1}, \ldots \mathbf{R}_{M}\right) . \Psi_{m}(\mathbf{x}, \mathbf{Q})$ is the state function representing state $|m\rangle$. The state function is a solution to a many-particle stationary-state Schrödinger equation, the Hamiltonian of which includes the kinetic energy of the nuclei. $\Psi_{m}(\mathbf{x}, \mathbf{Q})$ is chosen to normalize to unity. 
For a canonical ensemble, the probability of being in a state $|m\rangle$ is given by the Boltzmann factor

$$
W_{m} \equiv \exp \left(-E_{m} / k T\right) / \sum_{i} \exp \left(-E_{i} / k T\right),
$$

in which $E_{m}$ is the energy of state $|m\rangle$ of the crystal. The summation extends over all states included in the canonical ensemble representing the crystal.

The canonical electron density is then given by

$$
\overline{\rho(\mathbf{r})}=\sum_{m} W_{m} \rho_{m}(\mathbf{r}) .
$$

In a previous paper on the vibrational average of X-ray scattering intensities (Stewart, 1977), the formal difference was noted between the procedure in which the Boltzmann weighting factor is applied to the electron density $\rho_{m}$ and the one in which this factor is applied to the intensity of the X-rays scattered elastically by the crystal in a particular quantum state. At that time there appeared no simple relation between the two expressions, neither could the relation to observable quantities be firmly established.

The present paper is a theoretical investigation on relations between molecular and crystal vibrational states and $\mathrm{X}$-ray scattering. It will be shown that the usual procedure in which the intensity of elastic X-ray scattering is related to the thermal-average electron density is formally incorrect, but of little consequence for a macroscopic system.

We restrict the discussion to kinematic scattering where the $\mathrm{X}$-ray photon undergoes a single scattering process by the target before being measured.

\section{Total and elastic $\mathbf{X}$-ray scattering}

In the X-ray scattering process, relatively weak interaction takes place between a radiation field and the scattering system.

Without interaction, the radiation field consists of the beam of photons emerging from the monochromator, all with the same direction and energy. The scattering system of electrons and nuclei is in a state $|m\rangle$. With interaction, the radiation field will still mainly consist of the primary beam, but photons proceeding in other directions appear. If the scattering system remains in the same energy state, the scattered photons will have the same energy as the incident photons and the scattering is called elastic. In general, elastic scattering is accompanied by inelastic scattering in which the scattering system has gone to another energy state.

In the case of weak interaction, the first Born approximation suffices to describe the process. This theory, known by crystallographers as kinematic scattering, leads to Fermi's Golden Rule (Alonso \& Valk, 1973), which forms the basis of our discussion:

$$
W=(2 \pi / h)\left|\left\langle f\left|\mathscr{O}^{\prime}\right| i\right\rangle\right|^{2} \rho(\omega) \Delta \omega \Delta \Omega .
$$

$W$ is the probability that in a unit of time a transition takes place from the initial state $|i\rangle$ to any of the final states $|f\rangle$, whereby energy is conserved. The initial state $|i\rangle$ can be represented by $\left|m, \mathbf{k}_{0}\right\rangle$ in which $|m\rangle$ describes the scattering system and $\mathbf{k}_{0}$ is the wave vector of the incident beam $\left[\left|\mathbf{k}_{0}\right|=\left(2 \pi / \lambda_{0}\right)\right]$. The final states $|f\rangle$ are described similarly by $\left|n, \mathbf{k}_{1}\right\rangle$. Only those final states are included in which the scattered photons move in the direction of the counter and have an energy between $\omega$ and $\omega+\Delta \omega$. With a small counter opening, all these final states of the radiation field can be characterized by $\mathbf{k}_{1} . \rho(\omega) \Delta \omega \Delta \Omega$ gives the number of radiation modes in the solid angle $\Delta \Omega$ subtended by the counter and in the frequency range $\Delta \omega$ that is included in the measurement. $\mathscr{H}^{\prime}$ is the Hamiltonian describing the interaction between the X-rays and the scattering system.

By starting from this expression we do not include effects of extinction or anomalous scattering. The matrix term can be developed by introducing

$$
\mathscr{P}^{\prime}=\sum_{j}\left(e^{2} / 2 m\right) \mathbf{A}^{2}\left(\mathbf{r}_{j}\right),
$$

where $\mathbf{A}\left(\mathbf{r}_{j}\right)$ is the vector potential at the position of electron $j . e$ and $m$ are the electronic charge and mass respectively. This expression reflects the fact that only electrons contribute appreciably to the scattering of the $\mathrm{X}$-rays.

A discussion of the Hamiltonian and a derivation of the resulting expression can be found in Feil (1977). The transition probability $W$ is proportional to the intensity measured by the counter. The final expression for the intensity contains the (in)elastic form factors $F_{n m}(\mathbf{K})$,

$$
\begin{aligned}
F_{n m}(\mathbf{K}) \equiv & \sum_{j=1}^{L}\langle n| e^{+i \mathbf{K} \cdot \mathrm{r}_{j}|m\rangle} \\
= & \sum_{j=1}^{L} \int \Psi_{n}^{*}(\mathbf{x}, \mathbf{Q}) e^{+i \mathbf{K} \cdot \mathbf{r}_{j}} \\
& \quad \times \Psi_{m}(\mathbf{x}, \mathbf{Q}) \mathrm{d}^{4 L} \mathbf{x} \mathrm{d}^{4 M} \mathbf{Q} .
\end{aligned}
$$

Since in the first Born approximation the sign of the exponent of (7) does not play a role, it has been chosen so as to conform to the usage of most textbooks on $\mathrm{X}$-ray diffraction.

The intensity scattered into the counter by a scatterer in state $|m\rangle$ is then given by

$$
I_{\text {total }}^{(m)}=I_{0} \sigma \sum_{n}\left|F_{n m}(K)\right|^{2} .
$$

$I_{0}$ is the intensity of the incident beam; $\sigma$ is defined as $\left(e^{2} / 4 \pi \varepsilon_{0} m c^{2} r\right)^{2}\left(\mathbf{e}_{k_{0}} \cdot \mathbf{e}_{k_{1}}\right)^{2}$, in which $r$ is the distance from the scattering system to the counter, $\mathbf{e}_{k_{0}}$ and $\mathbf{e}_{k_{1}}$ are the directions of polarization of the incident beam and diffracted beam respectively. If we average over all 
directions of polarization, the result will be the usual polarization factor. All other terms have their defined or normal meaning. J. J. Thomson found $I_{0} \sigma$ to be the intensity scattered by a free electron according to classical electromagnetic theory (Thomson \& Thomson, 1933).

In general, the target is in thermal contact with its surroundings, in which case the ensemble average of scattering by different states $|m\rangle$ has to be taken,

$$
I_{\text {total }}=\sum_{m} W_{m} I_{\text {total }}^{(m)} .
$$

We incorporate the Thomson factor $\sigma$ and the intensity of the incident beam into the left hand side of (7) and write simply

$$
I_{\text {total }}=\sum_{m} W_{m} I_{\text {total }}^{(m)}=\sum_{m} W_{m} \sum_{n}\left|F_{n m}\right|^{2} .
$$

In elastic scattering the final state of the scattering system is equal to the initial state and we can write

$$
I_{\text {elastic }}=\sum_{m} W_{m}\left|F_{m m}\right|^{2}
$$

and, consequently,

$$
I_{\text {inelastic }}=\sum_{m} W_{m} \sum_{n \neq m}\left|F_{n m}\right|^{2} .
$$

\section{Relation between purely elastic and Bragg scattering}

The elastic form factor for state $m$ is given by (7) with $n=m$. The electron density for state $m$ is given by (3). So the elastic form factor is related to $\rho_{m}(\mathbf{r})$ by

$$
F_{m m}(\mathbf{K})=\int \rho_{m}(\mathbf{r}) e^{+i \mathbf{K} \cdot \mathbf{r}} \mathrm{d}^{3} \mathbf{r} .
$$

From the definition of $F_{\mathrm{Bragg}}(\mathbf{K})$, given by (1),

$$
F_{\text {Bragg }}(\mathbf{K})=\sum_{m} W_{m} F_{m m}(\mathbf{K}) .
$$

We see that $F_{\text {Bragg }}(K)$ is the thermal average of the elastic form factors. The thermally-weighted meansquare difference of (13) and (14) is a mean-square fluctuation intensity term,

$$
\begin{aligned}
\Delta I(\mathbf{K}) & =\sum_{m} W_{m}\left|F_{m m}(\mathbf{K})-F_{\mathrm{Bragg}}(\mathbf{K})\right|^{2} \\
& =\sum_{m} W_{m}\left|F_{m m}(\mathbf{K})\right|^{2}-\left|F_{\mathrm{Bragg}}(\mathbf{K})\right|^{2},
\end{aligned}
$$

where $\left(15^{\prime}\right)$ follows from (14) and the normalization of $W_{m}$ as given by (4). From (11) we rewrite $\left(15^{\prime}\right)$ as

$$
I_{\text {elastic }}(\mathbf{K})=I_{\text {Bragg }}(\mathbf{K})+\Delta I(\mathbf{K}) \text {. }
$$

We note from (15) that $\Delta I(\mathbf{K})$ is non-negative so that $I_{\text {elastic }}(\mathbf{K}) \geq I_{\text {Bragg }}(\mathbf{K})$. At $T=0$, the system is in the ground state and in this case $I_{\text {elastic }}=I_{\text {Bragg }}$.

We see from (16) that the elastic, kinematic scattering from a target corresponding with an ensemble that has appreciable populations of the various excited vibrational states is not the same as, but always larger than, scattering from the mean thermal chargedensity distribution. The arguments presented up to now are perfectly general and hold in any state system of electrons and nuclei for which the perturbation treatment in (6) is valid. That is, kinematic or single scattering of monochromatic X-rays is assumed.

When the number of particles of a system increases, the number of states within a certain energy range goes up as well. When the number of degrees of freedom, $N$, becomes very large, most members of the ensemble have properties that closely resemble the average properties of the ensemble (Hill, 1956).

In the same way, the fluctuations in the electrondensity distribution are expected to reduce in relative magnitude when the system increases in size. If this is true, $\Delta I$ can be neglected for a system as large as a crystal or crystallite used in X-ray diffraction. To explore this aspect of the problem, we give a detailed result for a scattering system in the harmonic approximation for nuclear motion.

\section{Purely elastic scattering in harmonic theory}

For our purpose it is useful to distinguish between vibrational and electronic states. We therefore treat the state functions of the system in the adiabatic approximation (Born \& Huang, 1954)

$$
\Psi_{m}(\mathbf{x}, \mathbf{Q})=\psi_{e}(\mathbf{x} ; \mathbf{R}) \chi_{m}(\mathbf{Q}) .
$$

We assume in the following that the system remains in the electronic ground state, since the fluctuations in energy in the case of thermal equilibrium are too small to allow for appreciable population of electronically excited states. The system is so large, or the temperature so high, that many vibrational states of the ensemble are appreciably populated. So in (17) $\psi_{e}$ is the ground-state electronic wavefunction, which parametrically depends on the positional parameters of the nuclei, R. $\chi_{m}(\mathbf{Q})$ are the vibrational wavefunctions, whereby the adiabatic potential for the nuclei is determined by the electronic energy and nuclear repulsion. The index $m$ now describes the vibrational states. The intensity expression in adiabatic theory can be found as follows. From (7) and (17),

$$
\begin{aligned}
F_{m m}=\int & \chi_{m}^{*}(\mathbf{Q})\left[\int \psi_{e}^{*}(\mathbf{x} ; \mathbf{R}) \sum_{j}^{L} e^{i \mathbf{K} \cdot \mathbf{r} J} \psi_{e}(\mathbf{x} ; \mathbf{R}) \mathrm{d}^{4 L} \mathbf{x}\right] \\
& \times \chi_{m}(\mathbf{Q}) \mathrm{d}^{4 M} \mathbf{Q} .
\end{aligned}
$$


Defining

$$
\begin{aligned}
F(\mathbf{K} ; \mathbf{R}) & \equiv \int \psi_{e}^{*}(\mathbf{x} ; \mathbf{R}) \sum_{j}^{L} e^{i \mathbf{K} \cdot \mathbf{r} /} \psi_{e}(\mathbf{x} ; \mathbf{R}) \mathrm{d}^{4 L} \mathbf{x} \\
& \equiv \int \rho(\mathbf{r} ; \mathbf{R}) e^{i \mathbf{K} \cdot \mathbf{r}} \mathrm{d}^{3} \mathbf{r},
\end{aligned}
$$

we can write

$$
F_{m m}=\int \chi_{m}^{*}(\mathbf{Q}) F(\mathbf{K} ; \mathbf{R}) \chi_{m}(\mathbf{Q}) \mathrm{d}^{4 M} \mathbf{Q} .
$$

With (11) and (14),

$$
\begin{aligned}
& I_{\text {elastic }}(\mathbf{K})=\sum_{m} W_{m}\left|\int \chi_{m}^{*}(\mathbf{Q}) F(\mathbf{K} ; \mathbf{R}) \chi_{m}(\mathbf{Q}) \mathrm{d}^{4 M} \mathbf{Q}\right|^{2},(20) \\
& I_{\text {Bragg }}(\mathbf{K})=\left|\sum_{m} W_{m} \int \chi_{m}^{*}(\mathbf{Q}) F(\mathbf{K} ; \mathbf{R}) \chi_{m}(\mathbf{Q}) \mathrm{d}^{4 M} \mathbf{Q}\right|^{2} \cdot(21)
\end{aligned}
$$

For completeness we include $I_{\text {total }}(\mathbf{K})$ from (10) where only scattering from the manifold of vibrational or phonon states is considered

$$
\begin{aligned}
I_{\text {total }}(\mathbf{K}) & =\sum_{m} W_{m} \sum_{n}\left|\int \chi_{n}^{*}(\mathbf{Q}) F(\mathbf{K} ; \mathbf{R}) \chi_{m}(\mathbf{Q}) \mathrm{d}^{4 M} \mathbf{Q}\right|^{2} \\
& =\sum_{m} W_{m} \int \chi_{m}^{*}(\mathbf{Q})|F(\mathbf{K} ; \mathbf{R})|^{2} \chi_{m}(\mathbf{Q}) \mathrm{d}^{4 M} \mathbf{Q} .(22)
\end{aligned}
$$

To obtain the last line use has been made of the closure relation.

Born (1942) has developed an explicit expression for (22) within the harmonic approximation. We will follow a similar procedure for evaluation of $(20)$ and $(21)$. To proceed we introduce the model of rigid pseudo-atoms. In this model the electron-density distribution is assumed to be the superposition of partial distributions, each of which is rigid with respect to the position of one of the nuclei:

$$
\rho(\mathbf{r} ; \mathbf{R})=\sum_{p=1}^{M} \rho_{p}\left(\mathbf{r}-\mathbf{R}_{p} ; \mathbf{R}^{e}\right) .
$$

The form factor for the partial distribution $\rho_{p}$ is given by

$$
f_{p}(\mathbf{K})=\int \rho_{p}\left(\mathbf{r} ; \mathbf{R}_{e}\right) e^{j \mathbf{K} \cdot \mathbf{r}} \mathrm{d}^{3} \mathbf{r} .
$$

We define the displacement $\mathbf{u}_{p}$ by

$$
\mathbf{R}_{p} \equiv \mathbf{R}_{p}^{e}+\mathbf{u}_{p}
$$

Equation (18) can now be written as

$$
\begin{aligned}
F(\mathbf{K}, \mathbf{R}) & =\sum_{p=1}^{M} f_{p}(\mathbf{K}) e^{i \mathbf{K} \cdot \mathbf{R}_{p}^{e}} e^{j \mathbf{K} \cdot \mathbf{u}_{p}} \\
& \equiv \sum_{p=1}^{M} F_{p}^{e}(\mathbf{K}) e^{i \mathbf{K} \cdot \mathbf{u}_{p}}
\end{aligned}
$$

in which $F_{p}^{e}(\mathbf{K})$ is defined implicitly.

In the harmonic approximation, the adiabatic potential for the nuclei is truncated at the quadratic term for a Taylor-series expansion about $\mathbf{R}_{\boldsymbol{e}}$.
With normal coordinates, $\xi_{q}$, the nuclear Hamiltonian can then be written:

$$
\hat{\mathscr{H}}(\xi)=\sum_{q=1}^{N} \hat{\mathscr{H}}\left(\xi_{q}\right)
$$

$N$ is the number of degrees of freedom involved in the vibrational motion. This enables us to write

$$
\chi_{m}(\mathrm{Q}(\xi))=\prod_{q=1}^{N} \chi_{q}^{(m)}\left(\xi_{q}\right),
$$

in which $\chi_{q}^{(m)}\left(\xi_{q}\right)$ is the state function describing the $q$ th harmonic oscillator when the scattering system is in vibration state $|m\rangle$,

$$
\chi_{q}^{(m)}\left(\xi_{q}\right)=\exp \left(-\frac{1}{2} \xi_{q}^{2}\right) H_{m_{q}}\left(\zeta_{q}\right) .
$$

In (26), $H_{m_{q}}\left(\xi_{q}\right)$ is a normalized Hermite polynomial of order $m$. Equation (24) implies that the coefficients of the linear transformation relating $\mathbf{u}_{p}$ to $\xi_{q}$ are given by (Willis \& Pryor, 1975)

$$
\mathbf{u}_{p}=m_{p}^{-1 / 2} \sum_{q=1}^{N} \mathbf{e}_{q}(p) \xi_{q},
$$

where the $\mathbf{e}_{q}(p)$ are normalized according to

$$
\sum_{p=1}^{M} \mathbf{e}_{q}^{+}(p) \cdot \mathbf{e}_{q^{\prime}}(p)=\delta_{q q^{\prime}}
$$

and $m_{p}$ is the mass of nucleus $p$. With the introduction of (27) into (23),

$$
F(\mathbf{K} ; \mathbf{R})=\sum_{p=1}^{M} F_{p}^{e}(\mathbf{K}) \prod_{q=1}^{N} \exp \left[i \mathbf{K} \cdot \mathbf{e}_{q}(p) \xi_{q} m_{p}^{-1 / 2}\right] .
$$

Inserting this result in (19) and using (26) we get

$$
\begin{aligned}
& F_{m m}=\sum_{p=1}^{M} F_{p}^{e}(\mathbf{K}) \prod_{q=1}^{N} \int \chi_{q}^{(m)}\left(\xi_{q}\right) \\
& \times \exp \left[i \mathbf{K} \cdot \mathbf{e}_{q}(p) \xi_{q} m_{p}^{-1 / 2}\right] \chi_{q}^{(m)}\left(\xi_{q}\right) \mathrm{d} \xi_{q} .
\end{aligned}
$$

The integration over Hermite functions was reported by Stewart (1977). The result of the introduction of (25) into (29) is

$$
F_{m m}=\sum_{p=1}^{M} F_{p}^{e}(\mathbf{K}) \prod_{q=1}^{N} \exp \left(-\frac{1}{2} x_{p q}\right) L_{m_{q}}\left(x_{p q}\right),
$$

where

$$
x_{p q}=\frac{\left|\mathbf{K} \cdot \mathbf{e}_{q}(p)\right|^{2}}{m_{p}} \frac{\hbar}{2 \omega_{q}} .
$$

$L_{m_{q}}$ in (30) is a Laguerre polynomial of order $m$ and degree 0 . Calculation of $I_{\text {total }}$, using (22), requires the calculation of the expectation value of $|F(\mathbf{K} . \mathbf{R})|^{2}$. The same procedure is followed and the result is similar in form to (30) [see Stewart, 1977, equation (46)].

The derivation of the final expression for the intensities can be found in the Appendix. 
For all three intensity expressions (20), (21) and (22) a Debye-Waller temperature factor for each pseudoatom emerges. We therefore introduce

$$
F_{p}(\mathbf{K}) \equiv F_{p}^{e}(\mathbf{K}) \exp \left(-\frac{1}{2} \mathbf{K}^{+} \mathscr{U}_{p} \mathbf{K}\right),
$$

where $\mathscr{U}_{p}$ is the tensor that describes the mean-square amplitude of motion for nucleus $p$ (see $A 11$ ).

The intensity expressions are

$$
\begin{gathered}
I_{\text {Bragg }}(\mathbf{K})=\sum_{p} \sum_{p^{\prime}} F_{p}^{*}(\mathbf{K}) F_{p^{\prime}}(\mathbf{K})=\left|\sum_{p} F_{p}(\mathbf{K})\right|^{2}, \\
I_{\text {elastic }}(\mathbf{K})=\sum_{p} \sum_{p^{\prime}} F_{p}^{*}(\mathbf{K}) F_{p^{\prime}}(\mathbf{K}) \prod_{q=1}^{N} I_{0}\left[\frac{\mathbf{K} \cdot \mathbf{e}_{q}(p)}{\sqrt{m_{p}}}\right. \\
\left.\quad \times \frac{\left(-\partial \bar{\varepsilon}_{q} / \partial \beta\right)^{1 / 2}}{\omega_{q}^{2}} \frac{\mathbf{K} \cdot \mathbf{e}_{q}\left(p^{\prime}\right)}{\sqrt{m_{p^{\prime}}}}\right], \\
I_{\text {total }}(\mathbf{K})=\sum_{p} \sum_{p^{\prime}} F_{p}(\mathbf{K}) F_{p}^{*}(\mathbf{K}) \prod_{q=1}^{N} \exp \left[\frac{\mathbf{K} \cdot \mathbf{e}_{q}(p)}{\sqrt{m_{p}}} \frac{\bar{\varepsilon}_{q}}{\omega_{q}^{2}}\right. \\
\left.\times \frac{\mathbf{K} \cdot \mathbf{e}_{q}\left(p^{\prime}\right)}{\sqrt{m_{p^{\prime}}}}\right] .
\end{gathered}
$$

In (34), $I_{0}(x)$ is a zeroth order modified Bessel function, $\beta$ is $(k T)^{-1}$ and $\bar{\varepsilon}_{q}$ is the mean thermal energy for normal mode, or oscillator, $q$. The explicit formulas for $\bar{\varepsilon}_{q}$ and the related function in (34) are

$$
\begin{aligned}
\bar{\varepsilon}_{q} & =\frac{1}{2} h \omega_{q} \operatorname{coth}\left(\hbar \omega_{q} / 2 k T\right), \\
\left(-\partial \bar{\varepsilon}_{q} / \partial \beta\right)^{1 / 2} & =\frac{1}{2} h \omega_{q} \operatorname{csch}\left(h \omega_{q} / 2 k T\right) .
\end{aligned}
$$

Note that the product in (35) can be closed into the Born (1942) scattering matrix $S_{p p^{\prime}}$.

For low $T\left(h \omega_{q} \gg k T\right),(37)$ goes to zero so that $I_{0}$ becomes unity and $I_{\text {elastic }} \simeq I_{\text {Bragg }}$. This feature of (34) confirms our conclusions from the general theory outlined in the previous section. At high $T$, for which $h \omega_{q} \ll k T$, (37) becomes (36). In this case,

$$
\begin{aligned}
I_{\text {elastlc }}(\mathbf{K})_{T \rightarrow \infty} & \simeq \sum_{p} \sum_{p^{\prime}} F_{p}^{*}(\mathbf{K}) F_{p^{\prime}}(\mathbf{K}) \\
\times & \prod_{q=1}^{N} I_{0}\left[\frac{\mathbf{K} \cdot \mathbf{e}_{q}(p)}{\sqrt{m_{p}}} \frac{\bar{\varepsilon}_{q}}{\omega_{q}^{2}} \frac{\mathbf{K} \cdot \mathbf{e}_{q}\left(p^{\prime}\right)}{\sqrt{m_{p^{\prime}}}}\right] .
\end{aligned}
$$

It is (38) which becomes interesting and we explore its behaviour for $N$ small and large.

\section{Elastic scattering by a small molecule and by a crystal}

We first consider an oriented diatomic molecule at a temperature for which (38) is applicable. The elastic scattering is detected when the energy resolution is sufficient to resolve the vibrational structure. For the single internal vibration the displacements of the atoms along the internuclear axis are given by (27):

$$
u_{1}=m_{1}^{-1 / 2}|\mathrm{e}(1)| \xi
$$

and

$$
u_{2}=-m_{2}^{-1 / 2}|\mathbf{e}(2)| \xi \text {. }
$$

During the vibration the centre of mass remains stationary:

$$
m_{1}^{1 / 2}|\mathbf{e}(1)|-m_{2}^{1 / 2}|\mathbf{e}(2)|=0 .
$$

The normalized eigenvectors satisfy (28):

$$
|\mathrm{e}(1)|^{2}+|\mathbf{e}(2)|^{2}=1 .
$$

Combination of (39) and (40) results in

$$
|\mathbf{e}(1)|=\left(\mu / m_{1}\right)^{1 / 2}
$$

and

$$
|\mathbf{e}(2)|=\left(\mu / m_{2}\right)^{1 / 2},
$$

in which $\mu$ is the reduced mass of the molecule.

The mean-square deviation from the equilibrium internuclear distance $|\mathbf{R}|$ is then found to be

$\overline{x_{12}^{2}} \equiv \overline{\left|u_{1}-u_{2}\right|^{2}}=\left(\mu^{1 / 2} / m_{1}+\mu^{1 / 2} / m_{2}\right)^{2} \overline{\xi^{2}}=\mu^{-1} \bar{\varepsilon} / \omega^{2}$

With (41), we write

$$
\frac{\mathbf{K} \cdot \mathbf{e}(1)}{\sqrt{m_{1}}}=\frac{\mu^{1 / 2}}{m_{1}}|\mathbf{K}| \eta
$$

and

$$
\frac{\mathbf{K} . \mathbf{e}(2)}{\sqrt{m_{2}}}=\frac{\mu^{1 / 2}}{m_{2}}|\mathbf{K}| \eta,
$$

where $\eta$ is the direction cosine between the scattering vector $\mathbf{K}$ and the internuclear axis.

If we take $m_{1}=m_{2}$ for simplicity, (38) can be written for our diatomic molecule with a single vibration

$$
\begin{aligned}
& I_{\text {elastic }}(\mathbf{K})=I_{\text {Bragg }}(\mathbf{K}) I_{0}\left(\frac{1}{4} \overline{x_{12}^{2}} K^{2} \eta^{2}\right), \\
& I_{\text {Bragg }}(\mathbf{K})=\left|F_{1}(\mathbf{K})+F_{2}(\mathbf{K})\right|^{2},
\end{aligned}
$$

in which use has been made of (33).

For $\mathbf{K}$ parallel to $\mathbf{R}(\eta=1)$, the effect will be largest. When $\frac{1}{4} \overline{x_{12}^{2}} K^{2} \eta^{2}=1, I_{0}$ in $(43)$ is $1.27 . I_{\text {elastic }}(\mathbf{K})$ is then $27 \%$ larger than Bragg scattering. The example given here serves to illustrate that for a small molecule with a few normal modes at a temperature with $k T \simeq$ $h \omega_{q}$, the elastic and the Bragg scattering intensities are significantly different.

We now turn to (38), the high-temperature form of $I_{\text {elastic }}$, for the case of a large molecule, e.g. a crystal. The $\bar{\varepsilon}_{q} /\left(\omega_{q}^{2} \sqrt{m_{p} m_{p^{\prime}}}\right)$ in the argument of the Bessel function is the contribution by mode $q$ to the mean-square deviation from the equilibrium distance 
between atoms $p$ and $p^{\prime}, \overline{\left|\mathbf{u}_{p}-\mathbf{u}_{p^{\prime}}\right|^{2}} \equiv \overline{x_{p p^{\prime}}^{2}}$. We seek that mode $q_{0}$ for which $\left(\overline{x_{p p^{\prime}}}\right)_{q_{0}}$ is a maximum. Then, $\left|\mathbf{e}_{q}(p)\right| \simeq N^{-1 / 2}$ with $N=3 M$, and

$$
\begin{aligned}
\prod_{q=1}^{N} & I_{0}\left(\frac{\mathbf{K} \cdot \mathbf{e}_{q}(p)}{\sqrt{m_{p}}} \frac{\bar{\varepsilon}_{q}}{\omega_{q}^{2}} \frac{\mathbf{K} \cdot \mathbf{e}_{q}\left(p^{\prime}\right)}{\sqrt{m_{p^{\prime}}}}\right) \\
& <\prod_{q=1}^{N} I_{0}\left[|\mathbf{K}|^{2}\left(\overline{x_{p p^{\prime}}}\right)_{q_{0}} / N\right] \\
& =\left\{I_{0}\left[|K|^{2}\left(\overline{x_{p p^{\prime}}^{2}}\right)_{q_{0}} / N\right]\right\}^{N} \\
& =\left\{1+\frac{1}{4}\left[|K|^{2}\left(\overline{x_{p p^{\prime}}^{2}}\right)_{q_{0}} / N\right]^{2}+\ldots\right\}^{N} .
\end{aligned}
$$

For $N$ large, (45) is $1+\frac{1}{4}\left[|\mathbf{K}|^{2}\left(\overline{x_{p p^{\prime}}}\right)_{q_{0}}\right]^{2} / N$ and $I_{\text {elastic }}(\mathbf{K})$ approaches $I_{\text {Bragg }}(\mathbf{K})$. Thus we see from the reasoning here that $\Delta I / I_{\mathrm{Bragg}} \simeq O\left(N^{-1}\right)$ for $N$ large. So for even a very small crystal with $10^{6}$ normal modes of vibration, the relative difference between $I_{\text {elastic }}$ and $I_{\text {Bragg }}$ at high temperature is $10^{-6}$. This is not a measurable difference.

\section{Conclusion}

For a large collection of atoms in a crystal, purely elastic X-ray scattering intensities can be properly interpreted as kinematic scattering by a thermally averaged charge-density distribution function as long as the first Born approximation does not break down. For gas-phase scattering by small molecules at high temperature the 'elastic' scattering (resolved to within vibrational shifts) can be significantly different from Bragg scattering. It is emphasized, however, that reduction of the elastic intensity to quantum-mechanical charge densities for the equilibrium nuclear distribution cannot in general be accomplished with harmonic vibration theory as is done in this paper. (Moreover, to emphasize a 'conservation' of difficulty we point out that as $N$ gets large, the first Born approximation breaks down.) The problem of dynamical X-ray scattering by a perfect crystal in thermal equilibrium with its environment has been neither explored nor alluded to in this paper.

RFS acknowledges support by the Netherlands Organization for Advancement of Pure Research (ZWO) and by NSF Grant CHE-77-09649.

\section{APPENDIX}

\section{Sums over Boltzmann-weighted series}

The following thermal averages have to be calculated:

$$
I_{\text {elastic }}=\sum_{m=0}^{\infty} W_{m}\left|F_{m m}\right|^{2},
$$

$$
I_{\mathrm{Bragg}}=\left|\sum_{m} W_{m} F_{m m}\right|^{2},
$$

in which (2) and (14) have been combined. For our particular model we found $F_{m m}$ to be

$$
F_{m m}=\sum_{p} F_{p}^{e}(\mathbf{K}) \prod_{q=1}^{N} \exp \left(-\frac{1}{2} x_{p q}\right) L_{m}\left(x_{p q}\right)
$$

and $W_{m}$ is given by (4).

The $E_{m}$ in (4) for a system of independent harmonic oscillators are

$$
E_{m}=\sum_{q=1}^{N} \varepsilon_{q}^{(m)}
$$

where

$$
\varepsilon_{q}^{(m)}=\hbar \omega_{q}\left(n_{q}^{(m)}+\frac{1}{2}\right) \equiv \hbar \omega_{q}\left(m_{q}+\frac{1}{2}\right) \equiv \varepsilon_{q} m_{q}
$$

and

$$
W_{m}=\prod_{q=1}^{N} W_{q}^{(m)}
$$

where

$$
W_{q}^{(m)}=\frac{\exp \left(-\varepsilon_{q} m_{q} / k T\right)}{\sum_{m_{q}}^{\infty} \exp \left(-\varepsilon_{q} m_{q} / k T\right)} .
$$

Let $Z_{q}=\exp \left(-h \omega_{q} / k T\right)$ so that

$$
W_{q}^{(m)}=Z_{q}^{m_{q}} / \sum_{n=0}^{\infty} Z_{q}^{n} \text {. }
$$

Since $Z_{q}<1$,

$$
W_{q}^{(m)}=\left(1-Z_{q}\right) Z_{q}^{m_{q}}
$$

From $(A 1),(30),(A 2)$ and $(A 3)$,

$$
\begin{gathered}
I_{\text {Bragg }}(\mathbf{K})=\mid \sum_{p} F_{p}^{e}(\mathbf{K}) \prod_{q=1}^{N}\left(1-Z_{q}\right) e^{-\frac{1}{2} x_{p q}} \\
\times\left.\sum_{m_{q}}^{\infty} Z_{q}^{m_{q}} L_{m_{q}}\left(x_{p q}\right)\right|^{2}
\end{gathered}
$$

and from (11),.(30), (A2) and (A3),

$$
\begin{aligned}
I_{\text {elastic }}=\sum_{p} & \sum_{p^{\prime}} F_{p}^{e} F_{p^{\prime}}^{e *} \prod_{q=1}^{N}\left(1-Z_{q}\right) e^{-\frac{1}{4} x_{p q}} e^{-\frac{1}{4} x_{p^{\prime} q}} \\
& \times \sum_{m_{q}}^{\infty} z_{q}^{m_{q}} L_{m_{q}}\left(x_{p q}\right) L_{m_{q}}\left(x_{p^{\prime} q}\right) .
\end{aligned}
$$

The sums over $m_{q}$ for each normal mode $q$ can be closed with the Hille-Hardy formula (Myller-Lebedeff, 
1907; see also Higher Transcendental Functions, 1953):

$$
\begin{aligned}
\sum_{n=0}^{\infty} & \frac{n !}{\Gamma(n+\alpha+1)} L_{n}^{\alpha}(x) L_{n}^{\alpha}(y) z^{n} \\
= & (1-z)^{-1} \exp \left[-\frac{z(x+y)}{(1-z)}\right](x y z)^{-1 / 2 \alpha} \\
& \quad \times I_{\alpha}\left[\frac{2(x y z)^{1 / 2}}{(1-z)}\right]
\end{aligned}
$$

where $I_{\alpha}$ is a modified Bessel function of order $\alpha$. For both $(A 4)$ and $(A 5), \alpha$ is zero. For $(A 4)$ we take $y=0$ so that $L_{n}(0)=I_{0}(0)=1$. Thus,

$$
\sum_{m_{q}}^{\infty} Z_{q}^{m_{q}} L_{m_{q}}\left(x_{p q}\right)=\left(1-Z_{q}\right)^{-1} \exp \left[-x_{p q} Z_{q} /\left(1-Z_{q}\right)\right]
$$

and

$$
\begin{aligned}
\sum_{m_{q}}^{\infty} Z_{q}^{m_{q}} L_{m_{q}}\left(x_{p q}\right) L_{m_{q}}\left(x_{p^{\prime} q}\right) \\
=\left(1-Z_{q}\right)^{-1} \exp \left[-Z_{q}\left(x_{p q}+x_{p^{\prime} q}\right) /\left(1-Z_{q}\right)\right] \\
\quad \times I_{0}\left[\frac{2\left(x_{p q} x_{p^{\prime} q} Z_{q}\right)^{1 / 2}}{1-Z_{q}}\right]
\end{aligned}
$$

When $(A 7)$ is combined with the exponential function in $(A 4)$ the argument becomes

$$
-\frac{1}{2} x_{p q}\left(1+Z_{q}\right) /\left(1-Z_{q}\right)=-\frac{1}{2} x_{p q} \operatorname{coth}\left(\frac{1}{2} h \omega_{q} / k T\right)
$$

and from the definition given to $x_{p q}[(31)],(A 4)$ becomes,

$$
I_{\mathrm{Bragg}}(\mathbf{K})=\left|\sum_{p} F_{p}^{e}(\mathbf{K}) \prod_{q=1}^{N} \exp \left(-\frac{1}{2} \frac{\left|\mathbf{K} \cdot \mathbf{e}_{q}(p)\right|^{2}}{m_{p}} \frac{\bar{\varepsilon}_{q}}{\omega_{q}^{2}}\right)\right|^{2} .
$$

The $N$ product of exponentials in $(A 10)$ is an exponential of the sum of $3 N$ arguments. This sum can be written (Born, 1942)

$$
\sum_{q=1}^{N}\left(\frac{\mathbf{K} \cdot \mathbf{e}_{q}(p)}{\sqrt{m_{p}}} \frac{\bar{\varepsilon}_{q}}{\omega_{q}^{2}} \frac{\mathbf{e}_{q}(p) \cdot \mathbf{K}}{\sqrt{m_{p}}}\right)=\mathbf{K}^{+} \mathscr{U}_{p} \mathbf{K},
$$

where $\mathscr{U}_{p}$ in $(A 9)$ is the tensor for the mean-square amplitude of motion for nucleus $p$. This gives us (32) in the text.
A similar reasoning holds for combining the exponentials in $(A 8)$ with $(A 5)$. The argument in the modified Bessel function, $I_{0}$, of $(A 8)$ becomes

$$
\begin{aligned}
\frac{2\left(x_{p q} x_{p^{\prime} q} Z_{q}\right)^{1 / 2}}{1-Z_{q}}= & \frac{\mathbf{K} \cdot \mathbf{e}_{q}(p)}{\sqrt{m_{p}}} \frac{\frac{1}{2} h \omega_{q} \operatorname{csch}\left(\frac{1}{2} h \omega_{q} / k T\right)}{\omega_{q}^{2}} \\
& \times \frac{\mathbf{e}_{q}\left(p^{\prime}\right) \cdot \mathbf{K}}{\sqrt{m_{p^{\prime}}}} \\
= & \frac{\mathbf{K} \cdot \mathbf{e}_{q}(p)}{\sqrt{m_{p}}} \frac{\left(-\partial \bar{\varepsilon}_{q} / \partial \beta\right)^{1 / 2}}{\omega_{q}^{2}} \\
& \times \frac{\mathbf{e}_{q}\left(p^{\prime}\right) \cdot \mathbf{K}}{\sqrt{m_{p^{\prime}}}}, \\
\beta= & (k T)^{-1} .
\end{aligned}
$$

To our knowledge, the product $\prod_{q} I_{0}\left(t_{q}\right)$ cannot be expressed in closed form by some other known function. Thus $(A 10),(A 11)$ and $(A 12)$ give (33) and (34) in the text. A detailed derivation of (35) by the same methods was previously reported (Stewart, 1977).

\section{References}

Alonso, M. \& Valk, H. (1973). Quantum Mechanics, p. 369. London: Addison Wesley.

BORN, M. (1942). Rep. Prog. Phys. 9, 294-333.

Born, M. \& Huang, K. (1954). Dynamical Theory of Crystal Lattices, p. 406. Oxford Univ. Press.

Coppens, P. \& Stevens, E. (1977). Adv. Quantum Chem. $10,1-35$.

FeIL, D. (1977). Isr. J. Chem. 16, 103-110.

Higher Transcendental Functions (1953). Vol. II, edited by A. ERDĚLYI, p. 189. New York: McGraw-Hill.

HILL, T. L. (1956). Statistical Mechanics, p. 55. New York: McGraw Hill.

Isr. J. Chem. (1977). 16, 89-229.

MYLLER-LeBEDEFF, W. (1907). Math. Ann. 64, 388-416.

Phys. Scr. (1977). 15, 65-162.

StewART, R. F. (1977). Isr. J. Chem. 16, 137-143.

Thomson, J. J. \& Thomson, G. P. (1933). Conduction of Electricity through Gases, 3rd ed., Vol. II, pp. 257-260. Cambridge Univ. Press.

WILlis, B. T. M. \& PRYoR, A. W. (1975). Thermal Vibrations in Crystallography, p. 14. Cambridge Univ. Press.

Woolfson, M. M. (1970). X-ray Crystallography, p. 192. Cambridge Univ. Press. 